\title{
On Certain Subclass of Harmonic Starlike Functions
}

\author{
A. Y. Lashin \\ Department of Mathematics, Faculty of Science, Mansoura University, Mansoura 35516, Egypt \\ Correspondence should be addressed to A. Y. Lashin; aylashin@mans.edu.eg
}

Received 13 February 2014; Accepted 19 March 2014; Published 9 April 2014

Academic Editor: V. Ravichandran

Copyright (C) 2014 A. Y. Lashin. This is an open access article distributed under the Creative Commons Attribution License, which permits unrestricted use, distribution, and reproduction in any medium, provided the original work is properly cited.

Coefficient conditions, distortion bounds, extreme points, convolution, convex combinations, and neighborhoods for a new class of harmonic univalent functions in the open unit disc are investigated. Further, a class preserving integral operator and connections with various previously known results are briefly discussed.

\section{Introduction}

A continuous complex-valued function $f=u+i v$ is said to be harmonic in a simply connected domain $D \subset \mathbb{C}$ if both $u$ and $v$ are real harmonic in $D$. There is a close interrelation between analytic functions and harmonic functions. For example, for real harmonic functions $u$ and $v$, there exist analytic functions $U$ and $V$ so that $u=\operatorname{Re}(U)$ and $v=\operatorname{Im}(V)$. Then $f(z)=h(z)+\overline{g(z)}$, where $h$ and $g$ are, respectively, the analytic functions $(U+V) / 2$ and $(U-V) / 2$. In this case, the Jacobian of $f=h+\bar{g}$ is given by $J_{f}=\left|h^{\prime}(z)\right|^{2}-\left|g^{\prime}(z)\right|^{2}$. The mapping $z \rightarrow f(z)$ is orientation preserving and locally one-to-one in $D$ if and only if $J_{f}>0$ in $D$. The function $f=h+\bar{g}$ is said to be harmonic univalent in $D$ if the mapping $z \rightarrow f(z)$ is orientation preserving, harmonic, and one-to-one in $D$. We call $h$ the analytic part and $g$ the coanalytic part of $f$ (see Clunie and Sheil-Small [1]).

Denote by $\mathscr{H}$ the class of functions $f=h+\bar{g}$ that are harmonic univalent and orientation preserving in the open unit disk $\mathbb{U}=\{z:|z|<1\}$ for which $f(0)=f_{z}(0)-1=$ 0 . Then for $f=h+\bar{g} \in \mathscr{H}$, we may express the analytic functions $f$ and $g$ as

$$
\begin{gathered}
h(z)=z+\sum_{n=2}^{\infty} a_{n} z^{n}, \\
g(z)=\sum_{n=1}^{\infty} b_{n} z^{n}, \quad\left|b_{1}\right|<1 .
\end{gathered}
$$

Note that $\mathscr{H}$ reduces to the class $\mathcal{S}$ of normalized analytic univalent functions if the coanalytic part of its members is zero. For this class the function $f(z)$ may be expressed as

$$
f(z)=z+\sum_{n=2}^{\infty} a_{n} z^{n} .
$$

A function $f=h+\bar{g}$ with $h$ and $g$ given by (1) is said to be harmonic starlike of order $\beta(0 \leq \beta<1)$ for $|z|=r<1$, if

$$
\frac{\partial}{\partial \theta}\left(\arg f\left(r e^{i \theta}\right)\right)=\operatorname{Re}\left(\frac{z h^{\prime}(z)-\overline{z g^{\prime}(z)}}{h(z)+\overline{g(z)}}\right) \geq \beta .
$$

The class of all harmonic starlike functions of order $\beta$ is denoted by $\mathcal{S}_{H}^{*}(\beta)$ and extensively studied by Jahangiri [2]. The cases $\beta=0$ and $b_{1}=0$ were studied by Silverman and Silvia [3] and Silverman [4]. Other related works of the class $\mathscr{H}$ also appeared in [5-16].

Definition 1. Let $f=h+\bar{g}$ where $h$ and $g$ are given by (1). Let $0 \leq \beta<1$ and $\alpha \geq 0$. Then $f \in \mathcal{S}_{H}^{*}(\alpha, \beta)$ if and only if

$$
\begin{aligned}
& \operatorname{Re}\left(\frac{\alpha z^{2} h^{\prime \prime}(z)+z h^{\prime}(z)+\overline{\alpha z^{2} g^{\prime \prime}(z)+(2 \alpha-1) z g^{\prime}(z)}}{h(z)+\overline{g(z)}}\right) \\
& \quad \geq \beta .
\end{aligned}
$$

We note that for $\alpha=0$, the class $\mathcal{S}_{H}^{*}(\alpha, \beta)$ reduces to the class $\mathcal{S}_{H}^{*}(\beta)$. Further, if the coanalytic part $g(z)$ is zero, the 
class $\mathcal{S}_{H}^{*}(\alpha, \beta)$ reduces to the class $\mathscr{P}(\alpha, \beta)$ of functions $f \in \mathcal{S}$ which satisfy the condition

$$
\operatorname{Re}\left(\frac{\alpha z^{2} f^{\prime \prime}(z)+z f^{\prime}(z)}{f(z)}\right) \geq \beta
$$

for some $\beta(0 \leq \beta<1), \alpha \geq 0, f(z) / z \neq 0$ and $z \in \mathbb{U}$. Observe that the classes $\mathscr{P}(\alpha, \beta)$ and $\mathscr{P}(\alpha, 0)$ were introduced and studied by many authors and these include, for example, by Obradovic and Joshi [17], Padmanabhan [18], Li and Owa [19], Xu and Yang [20], Singh and Gupta [21], and Lashin [22]. We also note that for $\alpha=0$, the class $\mathscr{P}(0, \beta)$ was studied by Silverman [23].

\section{Main Results}

The first theorem of this section determines the sufficient coefficient condition for functions $f=h+\bar{g}$ to belong to the class $\mathcal{S}_{H}^{*}(\alpha, \beta)$. The following lemma obtained by Jahangiri is needed.

Lemma 2 (see [2, Theorem 1]). Let $f=h+\bar{g}$ with $h$ and $g$ of the form (1) and let

$$
\sum_{n=2}^{\infty} \frac{n-\beta}{1-\beta}\left|a_{n}\right|+\sum_{n=1}^{\infty} \frac{n+\beta}{1-\beta}\left|b_{n}\right| \leq 1,
$$

where $0 \leq \beta<1$. Then $f$ is harmonic, orientation preserving, and univalent in $\mathbb{U}$, and $f \in \mathcal{S}_{H}^{*}(\beta)$.

Theorem 3. Let $f=h+\bar{g}$ where $h$ and $g$ are of the form (1). If

$$
\begin{aligned}
& \sum_{n=2}^{\infty} \frac{\alpha n(n-1)+n-\beta}{1-\beta}\left|a_{n}\right| \\
& \quad+\sum_{n=1}^{\infty} \frac{\alpha n(n+1)+n+\beta}{1-\beta}\left|b_{n}\right| \leq 1
\end{aligned}
$$

for some $\beta,(0 \leq \beta<1)$ and $\alpha \geq 0$, then $f$ is harmonic, orientation preserving, and univalent in $\mathbb{U}$ and $f \in \mathcal{S}_{H}^{*}(\alpha, \beta)$.

Proof. Since $(n-\beta) \leq \alpha n(n-1)+n-\beta$ and $n+\beta \leq$ $\alpha n(n+1)+n+\beta,(n \geq 1)$, it follows from Lemma 2 that $f \in \mathcal{S}_{H}^{*}(\beta)$ and hence $f$ is harmonic, orientation preserving, and univalent in $\mathbb{U}$. Now, we only need to show that if (7) holds then

$$
\begin{aligned}
& \operatorname{Re}\left(\frac{\alpha z^{2} h^{\prime \prime}(z)+z h^{\prime}(z)+\overline{\alpha z^{2} g^{\prime \prime}(z)+(2 \alpha-1) z g^{\prime}(z)}}{h(z)+\overline{g(z)}}\right) \\
& \quad=\operatorname{Re} \frac{A(z)}{B(z)} \geq \beta .
\end{aligned}
$$

Using the fact that $\operatorname{Re}(w) \geq \beta$ if and only if $|1-\beta+w| \geq$ $|1+\beta-w|$, it suffices to show that

$$
|A(z)+(1-\beta) B(z)|-|A(z)-(1+\beta) B(z)| \geq 0,
$$

where

$$
\begin{gathered}
A(z)=\alpha z^{2} h^{\prime \prime}(z)+z h^{\prime}(z)+\overline{\alpha z^{2} g^{\prime \prime}(z)+(2 \alpha-1) z g^{\prime}(z),} \\
B(z)=h(z)+\overline{g(z)} .
\end{gathered}
$$

Substituting for $A(z)$ and $B(z)$ in (9), we obtain

$$
\begin{aligned}
& |A(z)+(1-\beta) B(z)|-|A(z)-(1+\beta) B(z)| \\
& =\mid(2-\beta) z+\sum_{n=2}^{\infty}((\alpha n+1)(n-1)+2-\beta) a_{n} z^{n} \\
& +\sum_{n=1}^{\infty}(\alpha n(n+1)-(n-1+\beta)) \overline{b_{n}} \overline{z^{n}} \\
& -\mid-\beta z+\sum_{n=2}^{\infty}((\alpha n+1)(n-1)-\beta) a_{n} z^{n} \\
& +\sum_{n=1}^{\infty}(\alpha n(n+1)-(n+1+\beta)) \bar{b}_{n} \bar{z}^{n} \\
& \geq(2-\beta)|z| \\
& -\sum_{n=2}^{\infty}((\alpha n+1)(n-1)+2-\beta)\left|a_{n}\right||z|^{n} \\
& -\sum_{n=1}^{\infty}|\alpha n(n+1)-(n-1+\beta)|\left|b_{n}\right||z|^{n}-\beta|z| \\
& -\sum_{n=2}^{\infty}((\alpha n+1)(n-1)-\beta)\left|a_{n}\right||z|^{n} \\
& -\sum_{n=1}^{\infty}|\alpha n(n+1)-(n+1+\beta)|\left|b_{n}\right||z|^{n} \\
& \geq 2(1-\beta)|z| \\
& -2 \sum_{n=2}^{\infty}(\alpha n(n-1)+n-\beta)\left|a_{n}\right||z|^{n} \\
& -2 \sum_{n=1}^{\infty}(\alpha n(n+1)+(n+\beta))\left|b_{n}\right||z|^{n} \\
& \geq 2(1-\beta)|z| \\
& \times\left(1-\sum_{n=2}^{\infty} \frac{\alpha n(n-1)+n-\beta}{1-\beta}\left|a_{n}\right|\right. \\
& \left.-\sum_{n=1}^{\infty} \frac{\alpha n(n+1)+(n+\beta)}{1-\beta}\left|b_{n}\right|\right) \geq 0,
\end{aligned}
$$

by the given condition (7). 
The harmonic function

$$
\begin{aligned}
f(z)= & z+\sum_{n=2}^{\infty} \frac{1-\beta}{\alpha n(n-1)+n-\beta} x_{n} z^{n} \\
& +\sum_{n=2}^{\infty} \frac{1-\beta}{\alpha n(n+1)+(n+\beta)} \bar{y}_{n} \bar{z}^{n},
\end{aligned}
$$

where $\sum_{n=2}^{\infty}\left|x_{n}\right|+\sum_{n=1}^{\infty}\left|y_{n}\right|=1$ shows that the coefficient bound given in (7) is sharp. The functions of the form (12) are in $\mathcal{S}_{H}^{*}(\alpha, \beta)$ since

$$
\begin{gathered}
\sum_{n=2}^{\infty} \frac{\alpha n(n-1)+n-\beta}{1-\beta}\left|a_{n}\right|+\sum_{n=1}^{\infty} \frac{\alpha n(n+1)+n+\beta}{1-\beta}\left|b_{n}\right| \\
=\sum_{n=2}^{\infty}\left|x_{n}\right|+\sum_{n=1}^{\infty}\left|y_{n}\right|=1 .
\end{gathered}
$$

Remark 4. Setting $g(z)=0$ in Theorem 3 yields the result obtained by Lashin [22, Theorem 2.1].

We denote by $\bar{\delta}_{H}^{*}(\alpha, \beta)$ the class of functions $f \in$ $\mathcal{S}_{H}^{*}(\alpha, \beta)$ whose coefficients satisfy the condition (7).

Theorem 5. Let $0 \leq \alpha_{1}<\alpha_{2}$ and $0 \leq \beta<1$. Then $\overline{\mathcal{S}}_{H}^{*}\left(\alpha_{2}, \beta\right) \subset$ $\overline{\mathcal{S}}_{H}^{*}\left(\alpha_{1}, \beta\right)$.

Proof. For $f \in \overline{\mathcal{S}}_{H}^{*}\left(\alpha_{2}, \beta\right)$, it follows from (7) that

$$
\begin{aligned}
& \sum_{n=2}^{\infty} \frac{\alpha_{1} n(n-1)+n-\beta}{1-\beta}\left|a_{n}\right|+\sum_{n=1}^{\infty} \frac{\alpha_{1} n(n+1)+n+\beta}{1-\beta}\left|b_{n}\right| \\
& <\sum_{n=2}^{\infty} \frac{\alpha_{2} n(n-1)+n-\beta}{1-\beta}\left|a_{n}\right| \\
& \quad+\sum_{n=1}^{\infty} \frac{\alpha_{2} n(n+1)+n+\beta}{1-\beta}\left|b_{n}\right| \leq 1 .
\end{aligned}
$$

Hence $f \in \overline{\mathcal{S}}_{H}^{*}\left(\alpha_{1}, \beta\right)$.

As a consequence of Theorem 5 , the functions in $\overline{\mathcal{S}}_{H}^{*}(\alpha, \beta)$ are starlike harmonic in $\mathbb{U}$.

Corollary 6. For $\alpha \geq 0$ and $0 \leq \beta<1, \overline{\mathcal{S}}_{H}^{*}(\alpha, \beta) \subset \mathcal{S}_{H}^{*}(\beta)$.

\section{Distortion Bounds and Extreme Points}

In this section, we obtain the distortion bounds and extreme points for functions in the class $\overline{\mathcal{S}}_{H}^{*}(\alpha, \beta)$.
Theorem 7. Let $f=h+\bar{g}$ where $h$ and $g$ are of the form (1) and $f \in \overline{\mathcal{S}}_{H}^{*}(\alpha, \beta)$. Then for $|z|=r<1$, we have

$$
\begin{aligned}
|f(z)| \leq & \left(1+\left|b_{1}\right|\right) r \\
& +\left(\frac{1-\beta}{2 \alpha+2-\beta}-\frac{2 \alpha+1+\beta}{2 \alpha+2-\beta}\left|b_{1}\right|\right) r^{2}, \\
|f(z)| \geq & \left(1-\left|b_{1}\right|\right) r \\
& -\left(\frac{1-\beta}{2 \alpha+2-\beta}-\frac{2 \alpha+1+\beta}{2 \alpha+2-\beta}\left|b_{1}\right|\right) r^{2},
\end{aligned}
$$

where

$$
\left|b_{1}\right| \leq \frac{1-\beta}{2 \alpha+1+\beta} .
$$

The result is sharp.

Proof. We shall prove the first inequality. Let $f \in \overline{\mathcal{S}}_{H}^{*}(\alpha, \beta)$. Then we have

$$
\begin{aligned}
|f(z)| \leq & \left(1+\left|b_{1}\right|\right) r+\sum_{n=2}^{\infty}\left(\left|a_{n}\right|+\left|b_{n}\right|\right) r^{n} \\
\leq & \left(1+\left|b_{1}\right|\right) r+r^{2} \sum_{n=2}^{\infty}\left(\left|a_{n}\right|+\left|b_{n}\right|\right) \\
= & \left(1+\left|b_{1}\right|\right) r+\frac{1-\beta}{2 \alpha+2-\beta} \\
& \times \sum_{n=2}^{\infty} \frac{2 \alpha+2-\beta}{1-\beta}\left(\left|a_{n}\right|+\left|b_{n}\right|\right) r^{2}
\end{aligned}
$$

and so

$$
\begin{aligned}
|f(z)| \leq & \left(1+\left|b_{1}\right|\right) r \\
& +\frac{1-\beta}{2 \alpha+2-\beta} \\
& \times \sum_{n=2}^{\infty}\left(\frac{\alpha n(n-1)+n-\beta}{1-\beta}\left|a_{n}\right|\right. \\
& \left.\quad+\frac{\alpha n(n+1)+n+\beta}{1-\beta}\left|b_{n}\right|\right) r^{2} \\
\leq & \left(1+\left|b_{1}\right|\right) r \quad \frac{1-\beta}{2 \alpha+2-\beta}\left(1-\frac{2 \alpha+1+\beta}{1-\beta}\left|b_{1}\right|\right) r^{2} \\
= & \left(1+\left|b_{1}\right|\right) r \quad \\
& +\left(\frac{1-\beta}{2 \alpha+2-\beta}-\frac{2 \alpha+1+\beta}{2 \alpha+2-\beta}\left|b_{1}\right|\right) r^{2} .
\end{aligned}
$$

The proof of the inequality (16) is similar to the proof of the inequality (15), thus we omit it. 
The upper bound given for $f \in \overline{\mathcal{S}}_{H}^{*}(\alpha, \beta)$ is sharp and the equality occurs for the function

$$
\begin{aligned}
f(z)= & z+\left|b_{1}\right| \bar{z} \\
& +\left(\frac{1-\beta}{2 \alpha+2-\beta}-\frac{2 \alpha+1+\beta}{2 \alpha+2-\beta}\left|b_{1}\right|\right) \bar{z}^{2} \quad(z=r),
\end{aligned}
$$

where $\left|b_{1}\right| \leq(1-\beta) /(2 \alpha+1+\beta)$. This completes the proof of Theorem 7 .

Now, we determine the extreme points of the closed convex hull of the class $\overline{\mathcal{S}}_{H}^{*}(\alpha, \beta)$ denoted by clco $\overline{\mathcal{S}}_{H}^{*}(\alpha, \beta)$.

Theorem 8. Let $f=h+\bar{g}$ where $h$ and $g$ are given by (1). Then $f \in \operatorname{clco} \overline{\mathcal{S}}_{H}^{*}(\alpha, \beta)$ if and only if

$$
f(z)=\sum_{n=1}^{\infty}\left(X_{n} h_{n}+Y_{n} g_{n}\right)
$$

where

$$
\begin{gathered}
h_{1}(z)=z, \\
h_{n}(z)=z+\frac{1-\beta}{\alpha n(n-1)+n-\beta} z^{n} \quad(n=2,3, \ldots) ; \\
g_{n}(z)=z+\frac{1-\beta}{\alpha n(n+1)+n+\beta} \bar{z}^{n} \quad(n=1,2,3, \ldots), \\
\sum_{n=1}^{\infty}\left(X_{n}+Y_{n}\right)=1, \quad X_{n} \geq 0, Y_{n} \geq 0 .
\end{gathered}
$$

In particular, the extreme points of the class $\overline{\mathcal{S}}_{H}^{*}(\alpha, \beta)$ are $\left\{h_{n}\right\}$ and $\left\{g_{n}\right\}$, respectively.

Proof. For a function $f$ of the form (21), we have

$$
\begin{aligned}
f(z)= & \sum_{n=1}^{\infty}\left(X_{n} h_{n}+Y_{n} g_{n}\right) \\
= & \sum_{n=1}^{\infty}\left(X_{n}+Y_{n}\right) z \\
& +\sum_{n=2}^{\infty} \frac{1-\beta}{\alpha n(n-1)+n-\beta} X_{n} z^{n} \\
& +\sum_{n=1}^{\infty} \frac{1-\beta}{\alpha n(n+1)+n+\beta} Y_{n} \bar{z}^{n} \\
= & z+\sum_{n=2}^{\infty} \frac{1-\beta}{\alpha n(n-1)+n-\beta} X_{n} z^{n} \\
& +\sum_{n=1}^{\infty} \frac{1-\beta}{\alpha n(n+1)+n+\beta} Y_{n} \bar{z}^{n} .
\end{aligned}
$$

But

$$
\begin{aligned}
\sum_{n=2}^{\infty} & \frac{\alpha n(n-1)+n-\beta}{1-\beta}\left(\frac{1-\beta}{\alpha n(n-1)+n-\beta} X_{n}\right) \\
& +\sum_{n=1}^{\infty} \frac{\alpha n(n+1)+n+\beta}{1-\beta}\left(\frac{1-\beta}{\alpha n(n+1)+n+\beta} Y_{n}\right) \\
= & \sum_{n=2}^{\infty} X_{n}+\sum_{n=1}^{\infty} Y_{n}=1-X_{1} \leq 1 .
\end{aligned}
$$

Thus $f \in \operatorname{clco} \overline{\mathcal{S}}_{H}^{*}(\alpha, \beta)$.

Conversely, suppose that $f \in \operatorname{clco} \overline{\mathcal{S}}_{H}^{*}(\alpha, \beta)$. Set

$$
X_{n}=\frac{\alpha n(n-1)+n-\beta}{1-\beta}\left|a_{n}\right| \quad(n=2,3, \ldots),
$$

$$
Y_{n}=\frac{\alpha n(n+1)+n+\beta}{1-\beta}\left|b_{n}\right| \quad(n=1,2,3, \ldots) \text {. }
$$

Then by the inequality (7), we have $0 \leq X_{n} \leq 1$ ( $n=$ $2,3, \ldots)$ and $0 \leq Y_{n} \leq 1(n=1,2, \ldots)$. Define $X_{1}=$ $1-\sum_{n=2}^{\infty} X_{n}-\sum_{n=1}^{\infty} Y_{n}$ and note that $X_{1} \geq 0$. Thus we obtain $f(z)=\sum_{n=1}^{\infty}\left(X_{n} h_{n}+Y_{n} g_{n}\right)$. This completes the proof of the theorem.

\section{Convolution and Convex Combinations}

For two harmonic functions

$$
\begin{aligned}
& f(z)=z+\sum_{n=2}^{\infty} a_{n} z^{n}+\sum_{n=1}^{\infty} \overline{b_{n}} \bar{z}^{n}, \\
& F(z)=z+\sum_{n=2}^{\infty} A_{n} z^{n}+\sum_{n=1}^{\infty} \overline{B_{n}} \bar{z}^{n}
\end{aligned}
$$

we define their convolution

$$
(f * F)(z)=z+\sum_{n=2}^{\infty} a_{n} A_{n} z^{n}+\sum_{n=1}^{\infty} \overline{b_{n} B_{n}} \bar{z}^{n} .
$$

Using this definition, we show that the class $\overline{\mathcal{S}}_{H}^{*}(\alpha, \beta)$ is closed under convolution.

Theorem 9. For $0 \leq \beta<1$ and $\alpha \geq 0$, let $f, F \in \overline{\mathcal{S}}_{H}^{*}(\alpha, \beta)$. Then $f * F \in \overline{\mathcal{S}}_{H}^{*}(\alpha, \beta)$. 
Proof. We note that $\left|A_{n}\right| \leq 1$ and $\left|B_{n}\right| \leq 1$. For the convolution $(f * F)$, we have

$$
\begin{aligned}
& \sum_{n=2}^{\infty} \frac{\alpha n(n-1)+n-\beta}{1-\beta}\left|A_{n} a_{n}\right| \\
& \quad+\sum_{n=1}^{\infty} \frac{\alpha n(n+1)+n+\beta}{1-\beta}\left|B_{n} b_{n}\right| \\
& \leq \sum_{n=2}^{\infty} \frac{\alpha n(n-1)+n-\beta}{1-\beta}\left|a_{n}\right| \\
& \quad+\sum_{n=1}^{\infty} \frac{\alpha n(n+1)+n+\beta}{1-\beta}\left|b_{n}\right| \leq 1 .
\end{aligned}
$$

Therefore $f * F \in \overline{\mathcal{S}}_{H}^{*}(\alpha, \beta)$.

We show that the class $\overline{\mathcal{S}}_{H}^{*}(\alpha, \beta)$ is closed under convex combination of its members.

Theorem 10. The class $\overline{\mathcal{S}}_{H}^{*}(\alpha, \beta)$ is closed under convex combination.

Proof. For $i=1,2,3, \ldots$, let $f_{i} \in \overline{\mathcal{S}}_{H}^{*}(\alpha, \beta)$ where $f_{i}(z)$ is given by

$$
f_{i}(z)=z+\sum_{n=2}^{\infty} a_{n i} z^{n}+\sum_{n=1}^{\infty} \bar{b}_{n i} \bar{z}^{n} .
$$

Then by (7), we have

$$
\begin{aligned}
\sum_{n=2}^{\infty} \frac{\alpha n(n-1)+n-\beta}{1-\beta}\left|a_{n i}\right| \\
\quad+\sum_{n=1}^{\infty} \frac{\alpha n(n+1)+n+\beta}{1-\beta}\left|b_{n i}\right| \leq 1 .
\end{aligned}
$$

For $\sum_{i=1}^{\infty} t_{i}=1,0 \leq t_{i} \leq 1$, the convex combination of $f_{i}$ may be written as

$$
\sum_{i=1}^{\infty} t_{i} f_{i}=z+\sum_{n=2}^{\infty}\left(\sum_{i=1}^{\infty} t_{i} a_{n i}\right) z^{n}+\sum_{n=1}^{\infty}\left(\sum_{i=1}^{\infty} t_{i} \overline{b_{n i}}\right) \bar{z}^{n} .
$$

Then by (7), we have

$$
\begin{aligned}
& \sum_{n=2}^{\infty} \frac{\alpha n(n-1)+n-\beta}{1-\beta}\left|\sum_{i=1}^{\infty} t_{i} a_{n i}\right| \\
& \quad+\sum_{n=1}^{\infty} \frac{\alpha n(n+1)+n+\beta}{1-\beta}\left|\sum_{i=1}^{\infty} t_{i} \bar{b}_{n i}\right| \\
& \leq \sum_{i=1}^{\infty} t_{i}\left(\sum_{n=2}^{\infty} \frac{\alpha n(n-1)+n-\beta}{1-\beta}\left|a_{n i}\right|\right. \\
& \left.\quad+\sum_{n=1}^{\infty} \frac{\alpha n(n+1)+n+\beta}{1-\beta}\left|b_{n i}\right|\right) \\
& \leq \sum_{i=1}^{\infty} t_{i}=1 .
\end{aligned}
$$

Therefore $\sum_{i=1}^{\infty} t_{i} f_{i} \in \overline{\mathcal{S}}_{H}^{*}(\alpha, \beta)$.

\section{Neighborhood Results}

Following the earlier investigations by Goodman [24], Ruscheweyh [25], Altintas et al. [26], and Porwal and Aouf [27], we define the $\delta$-neighborhood of function $f(z) \in \mathscr{H}$ by

$$
\begin{aligned}
N_{\delta}(f)= & \left\{F \in \mathscr{H}: F(z)=z+\sum_{n=2}^{\infty} A_{n} z^{n}+\sum_{n=1}^{\infty} \overline{B_{n}} \bar{z}^{n},\right. \\
& \left.\sum_{n=2}^{\infty} n\left|a_{n}-A_{n}\right|+\sum_{n=1}^{\infty} n\left|b_{n}-B_{n}\right| \leq \delta\right\} .
\end{aligned}
$$

In particular, for the identity function $e(z)=z$, we immediately have

$$
\begin{aligned}
N_{\delta}(e)=\{ & \left\{F \in \mathscr{H}: F(z)=z+\sum_{n=2}^{\infty} A_{n} z^{n}+\sum_{n=1}^{\infty} \bar{B}_{n} \bar{z}^{n},\right. \\
& \left.\sum_{n=2}^{\infty} n\left|A_{n}\right|+\sum_{n=1}^{\infty} n\left|B_{n}\right| \leq \delta\right\} .
\end{aligned}
$$

Theorem 11. $\overline{\mathcal{S}}_{H}^{*}(\alpha, \beta) \subseteq N_{\delta}(e)$, where

$$
\delta=\frac{(1-\beta)(2 \alpha+1)}{(\alpha+1)(2 \alpha+1-\beta)}
$$

Proof. Let $f \in \overline{\mathcal{S}}_{H}^{*}(\alpha, \beta)$. Then, in view of (7), since $\alpha n(n-$ $1)+n-\beta$ and $\alpha n(n+1)+n+\beta$ are increasing functions of $n(n \geq 1)$, we have

$$
\begin{aligned}
(2 \alpha+ & 1-\beta)\left(\sum_{n=2}^{\infty}\left|a_{n}\right|+\sum_{n=1}^{\infty}\left|b_{n}\right|\right) \\
\leq & (2 \alpha+2-\beta) \sum_{n=2}^{\infty}\left|a_{n}\right|+(2 \alpha+1+\beta) \sum_{n=1}^{\infty}\left|b_{n}\right| \\
\leq & \sum_{n=2}^{\infty}(\alpha n(n-1)+n-\beta)\left|a_{n}\right| \\
& +\sum_{n=1}^{\infty}(\alpha n(n+1)+n+\beta)\left|b_{n}\right| \leq(1-\beta),
\end{aligned}
$$

which yields

$$
\sum_{n=2}^{\infty}\left|a_{n}\right|+\sum_{n=1}^{\infty}\left|b_{n}\right| \leq \frac{1-\beta}{(2 \alpha+1-\beta)} .
$$


On the other hand, we also find from (7)

$$
\begin{aligned}
& \left.(\alpha+1) \sum_{n=2}^{\infty} n\left|a_{n}\right|-\beta \sum_{n=2}^{\infty}\left|a_{n}\right|\right) \\
& +\left((\alpha+1) \sum_{n=1}^{\infty} n\left|b_{n}\right|-\beta \sum_{n=1}^{\infty}\left|b_{n}\right|\right) \\
& \leq \sum_{n=2}^{\infty}((\alpha(n-1)+1) n-\beta)\left|a_{n}\right| \\
& \quad+\sum_{n=1}^{\infty}((\alpha(n+1)+1) n+\beta)\left|b_{n}\right| \\
& \leq(1-\beta) .
\end{aligned}
$$

From (37) and (38), we obtain

$$
\begin{aligned}
(\alpha+1) & \left(\sum_{n=2}^{\infty} n\left|a_{n}\right|+\sum_{n=1}^{\infty} n\left|b_{n}\right|\right) \\
\leq & (1-\beta)+\beta\left(\sum_{n=2}^{\infty}\left|a_{n}\right|+\sum_{n=1}^{\infty}\left|b_{n}\right|\right) \\
& =(1-\beta)+\beta \frac{1-\beta}{(2 \alpha+1-\beta)},
\end{aligned}
$$

which is equivalent to

$$
\sum_{n=2}^{\infty} n\left|a_{n}\right|+\sum_{n=1}^{\infty} n\left|b_{n}\right| \leq \frac{(1-\beta)(2 \alpha+1)}{(\alpha+1)(2 \alpha+1-\beta)}=\delta .
$$

\section{A Family of Class Preserving Integral Operator}

In this section, we consider the closure property of the class $\overline{\mathcal{S}}_{H}^{*}(\alpha, \beta)$ under the Bernardi integral operator $F(z)$, which is defined by

$$
\begin{array}{r}
F(z)=\frac{c+1}{z^{c}} \int_{0}^{z} t^{c-1} h(t) d t+\overline{\frac{c+1}{z^{c}} \int_{0}^{z} t^{c-1} g(t) d t} \\
(c>-1) .
\end{array}
$$

Theorem 12. Let $f=h+\bar{g}$ be in the class $\overline{\mathcal{S}}_{H}^{*}(\alpha, \beta)$, where $h$ and $g$ are given by (1). Then $F(z)$ defined by (41) also belongs to the class $\overline{\mathcal{S}}_{H}^{*}(\alpha, \beta)$.

Proof. From the representation of $F$, it follows that

$$
F(z)=z+\sum_{n=2}^{\infty} \frac{c+1}{c+n} a_{n} z^{n}+\sum_{n=1}^{\infty} \frac{c+1}{c+n} \bar{b}_{n} \bar{z}^{n}
$$

Now

$$
\begin{aligned}
\sum_{n=2}^{\infty} & \frac{\alpha}{}(n-1)+n-\beta \\
& \left.+\sum_{n=1}^{\infty} \frac{\alpha+\beta(n+1)+n+\beta}{c+n}\left|a_{n}\right|\right) \\
\leq & \left.\sum_{n=2}^{\infty} \frac{\alpha+\beta}{c+n}\left|b_{n}\right|\right) \\
& +\sum_{n=1}^{\infty} \frac{\alpha n(n+1)+n+\beta}{1-\beta}\left|b_{n}\right| \leq 1
\end{aligned}
$$

by (7). Thus $F(z) \in \overline{\mathcal{S}}_{H}^{*}(\alpha, \beta)$.

\section{Conflict of Interests}

The author declares that there is no conflict of interests regarding the publication of this paper.

\section{Acknowledgments}

The author would like to express his gratitude to Professor Dr. V. Ravichandran and the referees for their valuable comments which have essentially improved the presentation of this paper.

\section{References}

[1] J. Clunie and T. Sheil-Small, "Harmonic univalent functions," Annales Academiae Scientiarum Fennicae: Series A I. Mathematica, vol. 9, pp. 3-25, 1984.

[2] J. M. Jahangiri, "Harmonic functions starlike in the unit disk," Journal of Mathematical Analysis and Applications, vol. 235, no. 2, pp. 470-477, 1999.

[3] H. Silverman and E. M. Silvia, "Subclasses of harmonic univalent functions," The New Zealand Journal of Mathematics, vol. 28, no. 2, pp. 275-284, 1999.

[4] H. Silverman, "Harmonic univalent functions with negative coefficients," Journal of Mathematical Analysis and Applications, vol. 220, no. 1, pp. 283-289, 1998.

[5] O. P. Ahuja and J. M. Jahangiri, "A subclass of harmonic univalent functions," Journal of Natural Geometry, vol. 20, no. 1-2, pp. 45-56, 2001.

[6] R. M. Ali, B. A. Stephen, and K. G. Subramanian, "Subclasses of harmonic mappings defined by convolution," Applied Mathematics Letters, vol. 23, no. 10, pp. 1243-1247, 2010.

[7] R. M. El-Ashwah, M. K. Aouf, A. A. M. Hassan, and A. H. Hassan, "A unified representation of some starlike and convex harmonic functions with negative coefficients," Opuscula Mathematica, vol. 33, no. 2, pp. 273-281, 2013.

[8] B. A. Frasin, "Comprehensive family of harmonic univalent functions," SUT Journal of Mathematics, vol. 42, no. 1, pp. 145155, 2006.

[9] J. M. Jahangiri, "Coefficient bounds and univalence criteria for harmonic functions with negative coefficients," Annales Universitatis Mariae Curie-Skłodowska A, vol. 52, no. 2, pp. 5766, 1998. 
[10] J. M. Jahangiri, G. Murugusundaramoorthy, and K. Vijaya, "Starlikeness of harmonic functions defined by Ruscheweyh derivatives," Indian Academy of Mathematics, vol. 26, no. 1, pp. 191-200, 2004.

[11] A. Janteng and S. A. Halim, "Properties of harmonic functions which are convex of order $\beta$ with respect to symmetric points," Tamkang Journal of Mathematics, vol. 40, no. 1, pp. 31-39, 2009.

[12] Y. C. Kim, J. M. Jahangiri, and J. H. Choi, "Certain convex harmonic functions," International Journal of Mathematics and Mathematical Sciences, vol. 29, no. 8, pp. 459-465, 2002.

[13] N. Magesh and S. Mayilvaganan, "On a subclass of harmonic convex functions of complex order," International Journal of Mathematics and Mathematical Sciences, vol. 2012, Article ID 496731, 13 pages, 2012.

[14] S. Nagpal and V. Ravichandran, "A subclass of close-to-convex harmonic mappings," Complex Variables and Elliptic Equations, vol. 59, no. 2, pp. 204-216, 2014.

[15] S. Nagpal and V. Ravichandran, "A comprehensive class of harmonic functions deined by convolution and its connection with integral transforms and hypergeometric functions," Studia Universitatis Babeş-Bolyai Mathematica, vol. 59, no. 1, pp. 41-55, 2014.

[16] K. G. Subramanian, B. A. Stephen, and S. K. Lee, "Subclasses of multivalent harmonic mappings defined by convolution," Bulletin of the Malaysian Mathematical Sciences Society, vol. 35, no. 3, pp. 717-726, 2012.

[17] M. Obradovic and S. B. Joshi, "On certain classes of strongly starlike functions," Taiwanese Journal of Mathematics, vol. 2, no. 3, pp. 297-302, 1998.

[18] K. S. Padmanabhan, "On sufficient conditions for starlikeness," Indian Journal of Pure and Applied Mathematics, vol. 32, no. 4, pp. 543-550, 2001.

[19] J.-L. Li and S. Owa, "Sufficient conditions for starlikeness," Indian Journal of Pure and Applied Mathematics, vol. 33, no. 3, pp. 313-318, 2002.

[20] N. Xu and D. Yang, "Some criteria for starlikeness and strongly starlikeness," Bulletin of the Korean Mathematical Society, vol. 42, no. 3, pp. 579-590, 2005.

[21] S. Singh and S. Gupta, "First order differential subordinations and starlikeness of analytic maps in the unit disc," Kyungpook Mathematical Journal, vol. 45, no. 3, pp. 395-404, 2005.

[22] A. Y. Lashin, "On a certain subclass of starlike functions with negative coefficients," Journal of Inequalities in Pure and Applied Mathematics, vol. 10, no. 2, pp. 1-18, 2009.

[23] H. Silverman, "Partial sums of starlike and convex functions," Journal of Mathematical Analysis and Applications, vol. 209, no. 1, pp. 221-227, 1997.

[24] A. W. Goodman, "Univalent functions and nonanalytic curves," Proceedings of the American Mathematical Society, vol. 8, no. 3, pp. 598-601, 1957.

[25] S. Ruscheweyh, "Neighborhoods of univalent functions," Proceedings of the American Mathematical Society, vol. 81, no. 4, pp. 521-527, 1981.

[26] O. Altintas, Ö. Özkan, and H. M. Srivastava, "Neighborhoods of a class of analytic functions with negative coefficients," Applied Mathematics Letters, vol. 13, no. 3, pp. 63-67, 2000.

[27] S. Porwal and M. K. Aouf, "On a new subclass of harmonic univalent functions defined by fractional calculus operator," Journal of Fractional Calculus and Applications, vol. 4, no. 10, pp. 1-12, 2013. 


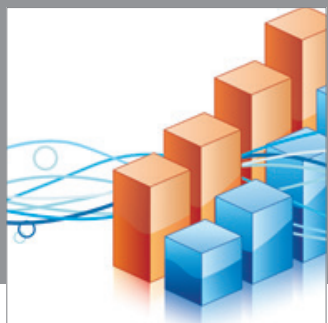

Advances in

Operations Research

mansans

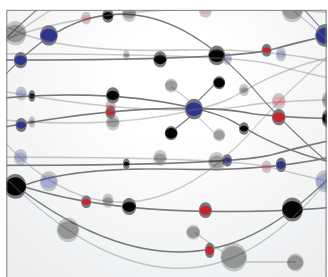

The Scientific World Journal
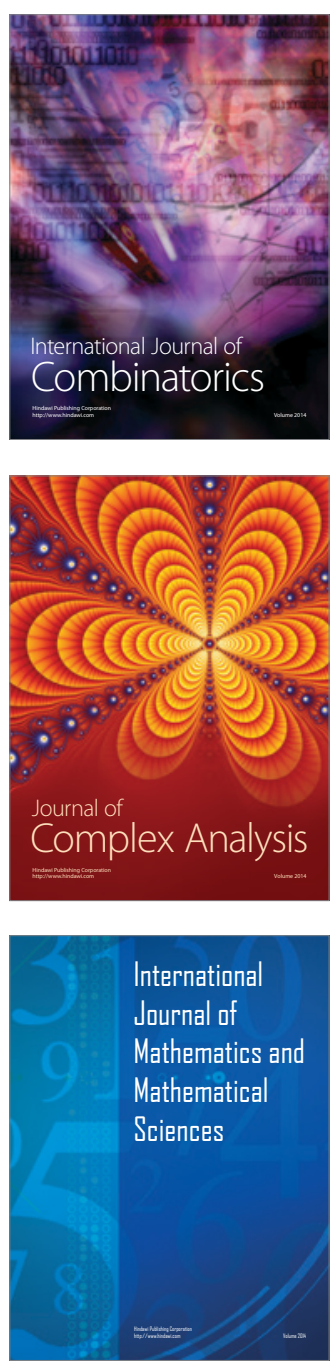
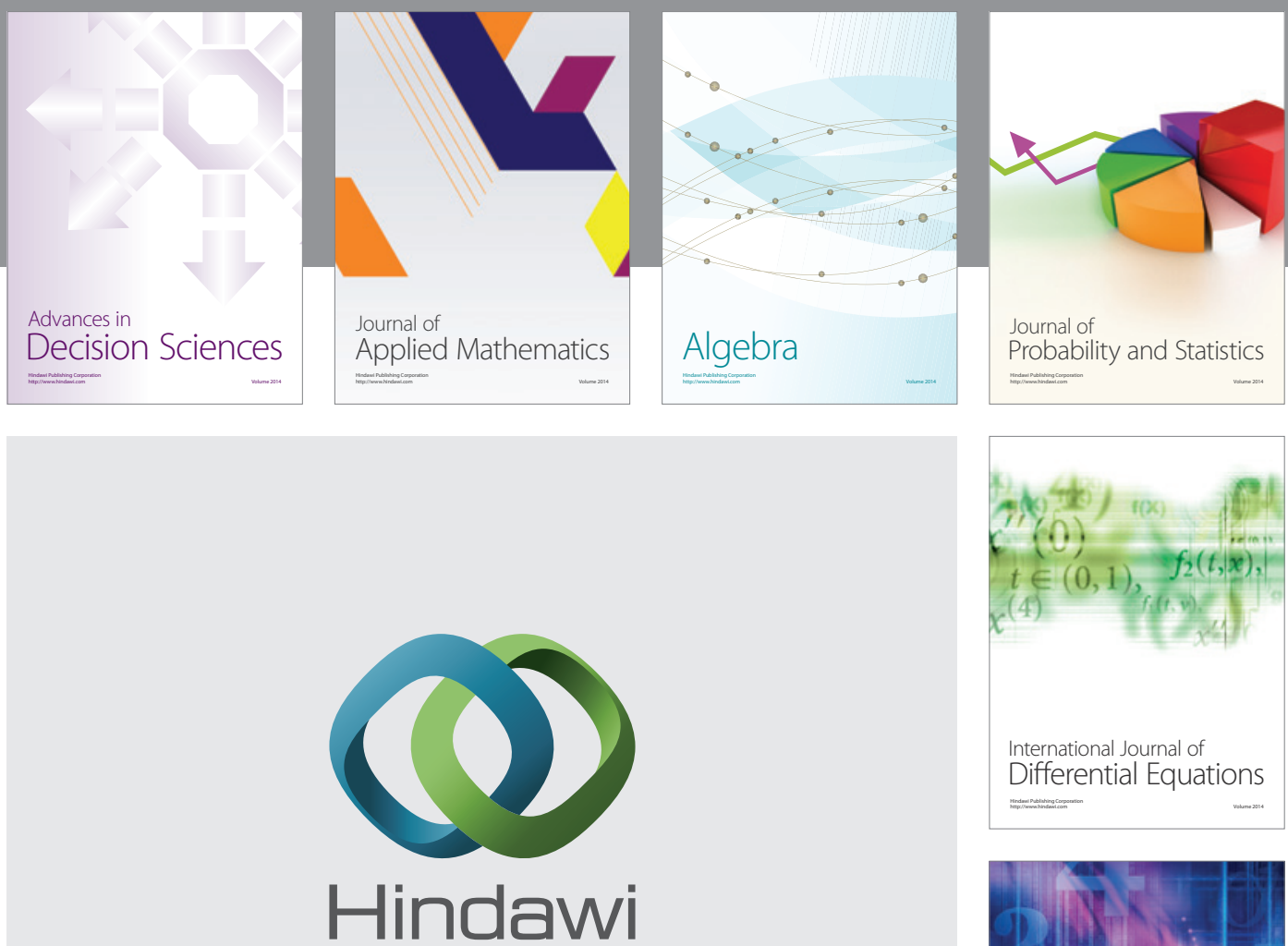

Submit your manuscripts at http://www.hindawi.com
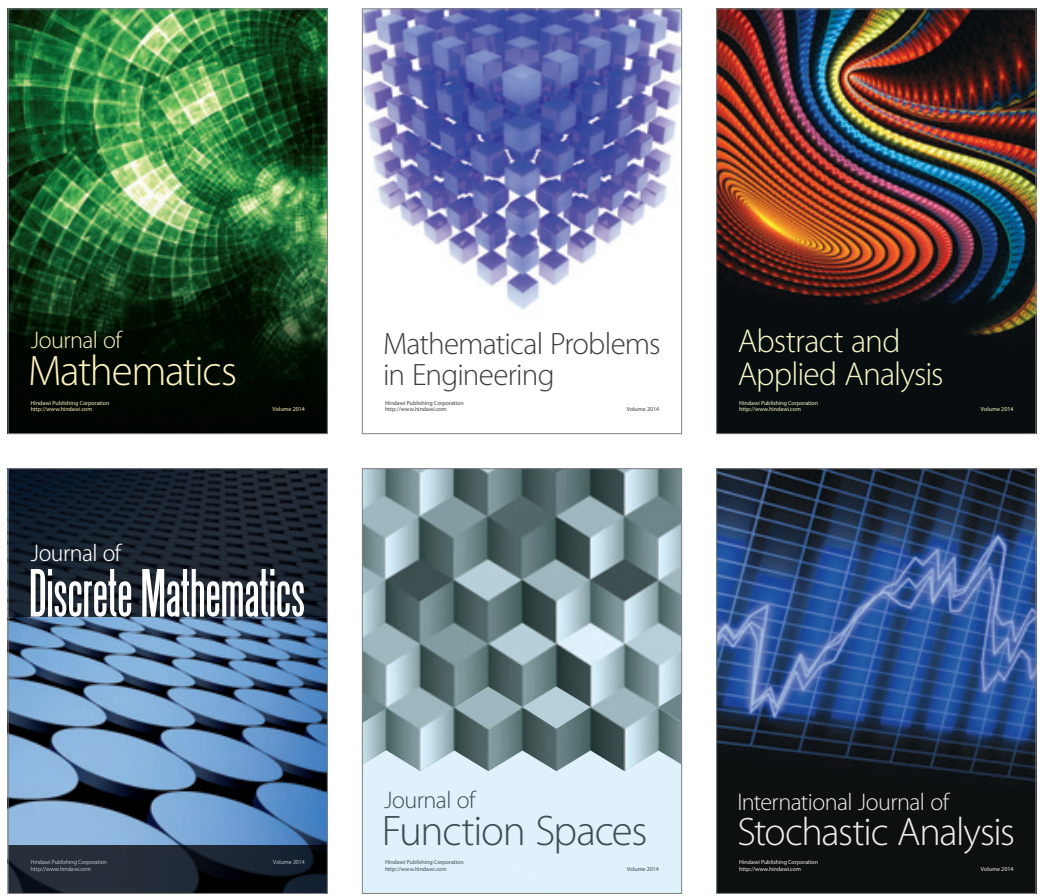

Journal of

Function Spaces

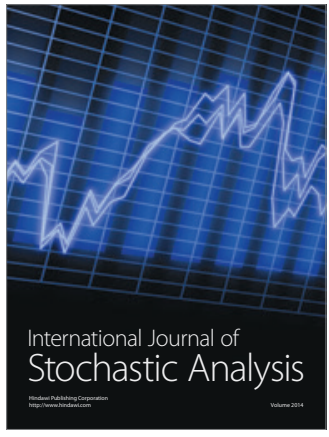

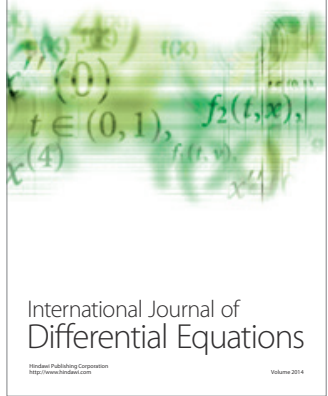
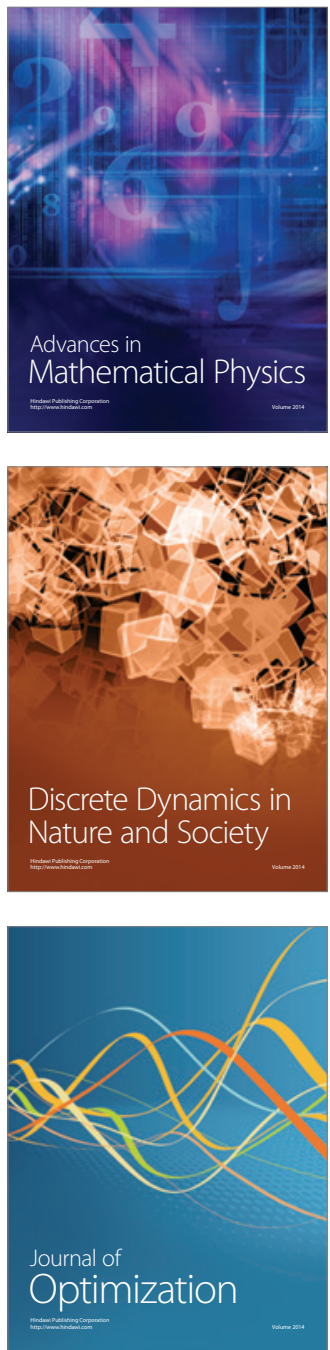